\title{
Crystal growth, Dielectric and Thermal Studies on N- Methyl-4-Nitroaniline: Isophthalic Acid Molecular Adduct Crystals
}

\author{
R. Lakshmi and P. Prabukanthan
}

\begin{abstract}
Organic compound N-methyl-4-nitroaniline isophthalic acid (NMNAIPA) needle shaped single crystals with a dimension of $4 \times 1 \times 1 \mathrm{~cm}^{3}$ were grown by slow evaporation technique in methanol as solvent at ambient temperature. The grown single crystals have been analyzed through powder $\mathrm{X}$ ray diffraction (XRD), proton nuclear magnetic resonance ( $\left.{ }^{1} \mathrm{HNMR}\right)$, carbon NMR $\left({ }^{13} \mathrm{CNMR}\right)$, Fourier transform infrared (FT-IR), Raman spectra and UV-Vis spectra. Based on powder XRD patterns, the as-grown NMNAIPA single crystal was a good crystalline in nature. The various planes of reflection have been identified from the powder XRD pattern. It belongs to the monoclinic crystal system. The presences of functional groups were identified from FT-IR and Raman spectra. UV-Vis-NIR spectral analysis showed $\mathbf{7 4 \%}$ transmission in the visible region for NMNAIPA crystal. The dielectric constant and dielectric loss of the as-grown NMNAIPA single crystal was studied as a function of frequency and temperature. Thermo gravimetric (TG) and differential scanning calorimetric (DSC) techniques were carried out to characterize the thermal behavior and stability of as-grown NMNAIPA single crystal. It has thermal stability around $421 \mathrm{~K}$. The nonlinear optical (NLO) activity test using a pulsed Nd:YAG laser confirms the generation of second harmonics and it shows $\mathbf{1 . 2 5}$ times relatively greater than KDP.
\end{abstract}

Index Terms-Dielectric Studies; Organic NLO Single Crystal; Synthesis; Spectral Studies; Thermal Property.

\section{INTRODUCTION}

The progress of new optical organic materials is of great interest due to their higher orders of optical transparencies than the conventional inorganic materials. Due to promising optical properties, the organic materials can be utilized in optical device fabrication which can replace the classical electronics, electronic switching circuits with organic materials [1-2]. The search for new advanced materials is an important area of current research in numerous disciplines of science and the development of many new technologies. The nonlinearity of organic compounds having a $\pi$-electron conjugated system is caused by nonlinear polarization that occurs due to the interaction between laser light which comprises a strong electromagnetic wave and the delocalized $\pi$-electrons in the organic molecule of interest.

In order to increase the magnitude of nonlinear polarization of the molecule, an electron donating group(donor)or an electron withdrawing group(acceptor)is

Published on September 11, 2017

R. Lakshmi and P. Prabukanthan are with Material Chemistry Lab, Department of Chemistry, Muthurangam Government Arts College, Vellore - 632 002, Tamil Nadu, India. (e-mail: laxmilogu11@gmail.com,pprabukanthan76@hotmail.com) introduced in to the $\pi$-electron conjugated system as a common technique for molecular design. Organic nonlinear optical (NLO) materials have attracted considerable interest owing to their large NLO properties, small dielectric constant, large electro-optic coefficient and short response time and its vital application in superconductors, semiconductors, photonic devices, electrical and magnetic fields. Dicarboxylic acids have been attracting immense interest due to their wide-ranging applications. Dicarboxylic acids exhibit many important properties; the two carboxylic groups can form linear structures by hydrogen bonds. The preparation of non-linear optical (NLO) crystals, the materials combine highly polarizable cations or molecules (i.e. carriers of non-linear properties) with hydrogenbonding organic acids providing thermal and structural stability [3].

In the present work an attempt has been made to grow $\mathrm{N}$ methyl-4-nitroaniline isophthalic acid (NMNAIPA) adduct single crystal by the slow evaporation method. The grown crystal (NMNAIPA) was subjected to various characterizations such as, Fourier transform infrared (FTIR), Raman study, nuclear magnetic resonance (NMR) analysis, optical, thermal analysis, dielectric studies and nonlinear optical studies.

\section{EXPERIMENT}

\section{A. Crystal growth}

The organic compound of N-methyl-4-nitroaniline isophthalic acid (NMNAIPA) single crystal was grown by slow evaporation solution growth method. N-methyl-4nitroaniline and isophthalic acid were procured from SigmaAldrich (USA) in analytical grade. It has used without further purification. Equimolar proportions of N-methyl-4nitroaniline and isophthalic acid was dissolved separately in methanol and the solutions were thoroughly mixed together using a mechanical stirrer for about 4 hours. The clear yellow solution obtained was filtered off using Whatman No. 40 grade filter paper to remove the suspended impurities. It was kept sideways without any mechanical shake for crystal growth in a dust free environment at ambient temperature. The formation of organic compound $\mathrm{N}$-methyl-4-nitroaniline isophthalic acid (NMNAIPA) single crystal reaction mechanism and chemical structure are illustrated in Fig. 1. Yellowish single crystals of NMNAIPA were collected carefully from mother liquor after four days. The harvested crystals were recrystallized repeatedly to get better-quality single crystals. The photograph of the single crystals of NMNAIPA is shown in Fig. 2. 


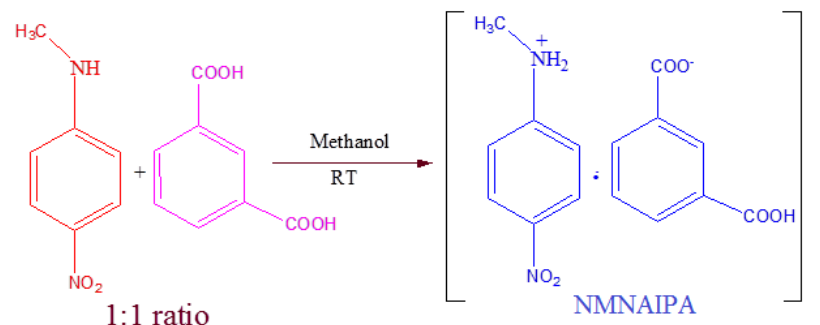

Fig.1. Reaction of NMNAIPA crystal

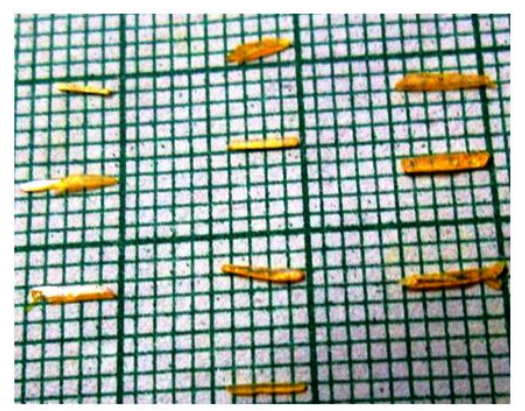

Fig. 2. Photograph of as-grown NMNAIPA crystal

\section{CHARACTERIZATION}

The grown crystals were subjected to various characterization studies. Powder X-ray diffraction patterns of the crystalline powder sample of NMNAIPA compound was recorded on a Rich-Seifert X-ray diffractometer. The room temperature mid-Fourier transform infrared spectra of NMNAIPA was recorded in the $400-4000 \mathrm{~cm}^{-1}$ at a resolution of $\pm 5 \mathrm{~cm}^{-1}$ by BRUKER spectrophotometer equipped with a $\mathrm{LiTaO}_{3}$ detector, a $\mathrm{KBr}$ beam splitter, a He$\mathrm{Ne}$ laser source and a boxcar atomization used for 250 averaged interferograms collected for both the sample and the background. $\mathrm{KBr}$ pellets contained a fine NMNAIPA powder obtained from the grown single crystals. The Raman spectrum was performed at room temperature using BRUKER-RFS 27 Raman spectrometer with laser $100 \mathrm{~mW}$. The UV- visible absorption spectrum of as grown NMNAIPA crystal was recorded in the wavelength region $200 \mathrm{~nm}$ to $1100 \mathrm{~nm}$ using a Lambda - 35spectrometer. The ${ }^{1} \mathrm{H}$ NMR and ${ }^{13} \mathrm{C}$ NMR spectrum of NMNAIPA dissolved in deutrated chloroform and spectrum was recorded using an advance III NMR spectrometer $400 \mathrm{MHz}$ instrument. The dielectric studies were carried out for HIOKI HITESTER model 3532-50 LCR meter and conventional two terminal sample holders in different frequency at different temperatures. The thermal behavior of the grown crystal was studied in TGA/DTA, 500 V20. 10 Build 36 analyzer in nitrogen atmosphere at a heating rate of $10^{\circ} \mathrm{C} / \mathrm{min}$ in the temperature ranging from $50^{\circ} \mathrm{C}$ to $300^{\circ} \mathrm{C}$. The crystal were illuminated using a spectra physics quanta Ray GCR-2(10) $\mathrm{Nd}$ :YAG laser using the first harmonics output of $1064 \mathrm{~nm}$ with a pulse energy of up to $300 \mathrm{~mJ}$. The non-linear optical (NLO) property of the grown NMNAIPA crystal was confirmed by Kurtz-Perry powder second harmonic generation (SHG) test.

\section{RESULTS AND DiSCUSSIONS}

\section{A. Powder XRD analysis}

The crystalline nature of the NMNAIPA grown crystals was confirmed by powder X-ray diffraction (XRD) analysis. Fig.3 (a-b) shows the powder XRD spectra of N-methyl-4nitroaniline (NMNA) and NMNAIPA compounds recorded at room temperature in a $2 \theta$ range $10^{\circ}$ to $90^{\circ}$ on with $\mathrm{CuK} \alpha$ $(1.5406 \mathrm{~nm})$ radiation. The unit cell parameters were calculated from the powder X-ray diffraction data using CRYSFIRE software. Lattice parameter values and hkl values were simulated and all in diffraction peaks were indexed. The cell parameters calculated from powder XRD data and the unit cell parameter values were $\mathrm{a}=9.107 \AA$, $\mathrm{b}$ $=10.613 \AA$ and $\mathrm{c}=8.142 \AA$, respectively. The results of sharp and well-defined diffraction peaks at specific $2 \theta$ angles testimonies the crystalline nature of the as-grown single crystals.

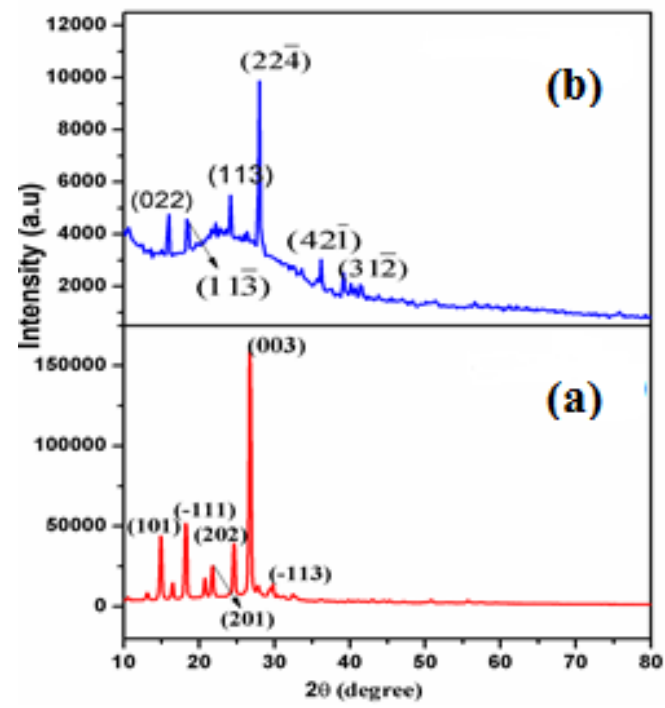

Fig. 3. Powder X-ray diffraction pattern of (a) NMNA and (b) NMNAIPA compound

\section{B. FTIR spectral analysis}

Vibrational spectroscopy is an important tool in understanding the chemical bonding and provides useful information to study the molecular structure of the synthesized compound. The Fourier transform infrared (FTIR) analysis was carried out to examine the presence of functional groups and their vibrational modes of the grown NMNAIPA crystal. The FT-IR spectra of NMNA and NMNAIPA are given in the Fig.4 (a-b). In the Fig. 4a, the peak observed at $3369 \mathrm{~cm}^{-1}$ assigned to $\mathrm{N}-\mathrm{H}$ stretching for the secondary amine group in NMNA. This peak cannot be followed in NMNAIPA compound (Fig.4b) due to the formation of primary amine with carboxylate anion. However, Fig. 4b, the peak appeared at $2654 \mathrm{~cm}^{-1}$ indicated that the withdrawing groups attached to isophthalic acid facilitate to liberate the proton of carboxylic group and transfer to the nitrogen atom of NMNA. The asymmetric and symmetric stretching vibrations of $\mathrm{COO}^{-}$groups are observed at 1679 and $1486 \mathrm{~cm}^{-1}$, respectively. The presence of asymmetric and symmetric stretching vibration of nitro group is confirmed by the peak at 1590 and $1295 \mathrm{~cm}^{-1}$, respectively. This nitro group does not change in the peak position of both NMNA and NMNAIPA compounds. The 
aromatic ring of $\mathrm{C}=\mathrm{C}$ is revealed at $1459 \mathrm{~cm}^{-1}$. The peaks at 830 and $920 \mathrm{~cm}^{-1}$ are due to $\mathrm{C}=\mathrm{C}-\mathrm{H}$ out of the plane.
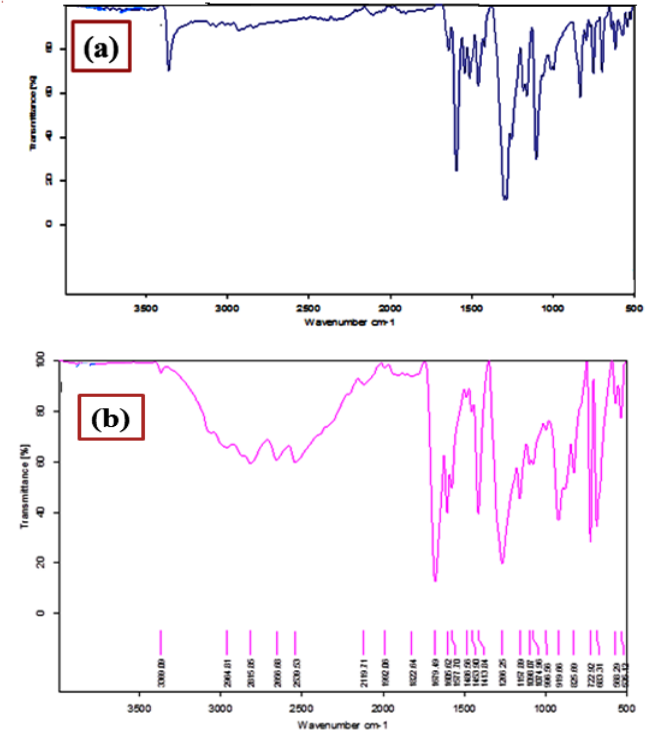

Fig .4. FT-IR spectra of (a) N-methyl-4-nitroaniline (NMNA) and (b) NMNAIPA crystal

\section{Raman spectrum study}

Raman activity tends to be a function of the covalent character of bonds. A Raman spectrum found to information about the backbone structure of the molecule, whereas the strong infrared features are indicative of polar segments [4]. Fig. 5 shows the FT-Raman spectra of NMNAIPA crystal. The less intense line due to $\mathrm{O}-\mathrm{H}$ stretching is observed at $3288 \mathrm{~cm}^{1}$. The peak appeared at $3082 \mathrm{~cm}^{-1}$ is due to aromatic $\mathrm{C}-\mathrm{H}$ stretching. The strong peak observed at 1633 $\mathrm{cm}^{-1}$ it's due to carbonyl $(\mathrm{C}=\mathrm{O})$ stretching in carboxylic acid. The peak appeared in 1435 and $1297 \mathrm{~cm}^{-1}$ it corresponding to asymmetric and symmetric stretching of nitro group. The peaks at 1602 and $1503 \mathrm{~cm}^{-1}$ it does correspond to aromatic ring skeletal vibrations. The peak appeared at $847 \mathrm{~cm}^{-1}$ other peak in lower wave numbers, regions are due to $\mathrm{C}-\mathrm{H}$ bending vibration modes.

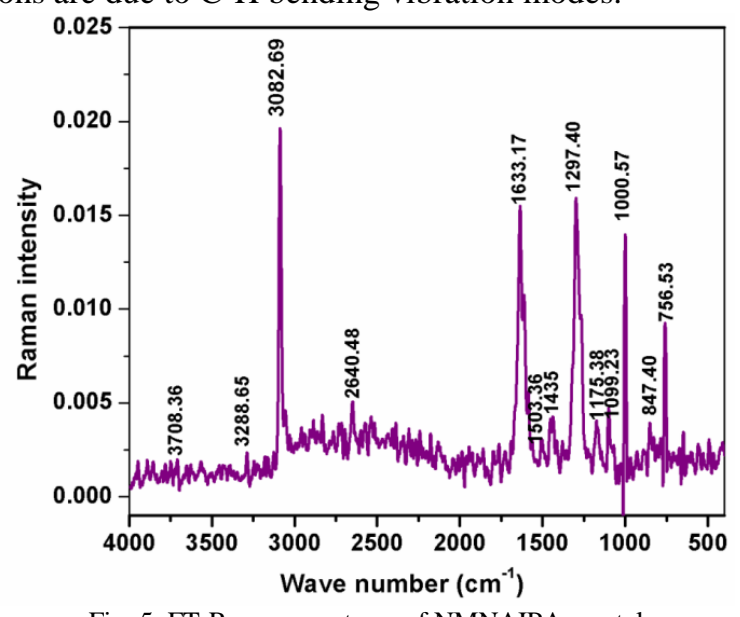

Fig. 5. FT-Raman spectrum of NMNAIPA crystal

\section{D. ${ }^{1} H$ NMR spectral analysis}

Proton nuclear magnetic resonance ( ${ }^{1} \mathrm{H}$ NMR) spectra recorded for NMNA and NMNAIPA are shown in Fig. 6 (ab). The NMNAIPA compound has 14 protons and it shows the signals at $\delta 13.31 \mathrm{ppm}(\mathrm{s}, 2 \mathrm{H}), \delta 8.48(\mathrm{~s}, 2 \mathrm{H})$ and $\delta 8.18$ $(\mathrm{m}, 4 \mathrm{H}), \delta 7.67(\mathrm{t}, 2 \mathrm{H}, J=8 \mathrm{~Hz})$ these signals corresponding to aromatic protons and it confirms the presence of three sets of chemically equivalent protons in the aromatic rings. The peak observed at $2.80 \mathrm{ppm}(\mathrm{d}, 1 \mathrm{H}, J=4 \mathrm{~Hz})$ shows doublet at aliphatic methyl proton. The broad singlet peak appeared at $\delta 3.36 \mathrm{ppm}(\mathrm{s}, 3 \mathrm{H})$ in $\mathrm{N}-\mathrm{H}$ proton in aromatic amine moiety. At an intermediate rate of exchange, the $\mathrm{NH}$ proton is partially decoupled, and a broad $\mathrm{NH}$ peak results. If the $\mathrm{NH}$ exchange rate is low, the $\mathrm{NH}$ peak is still broad because an electrical quadrupole moment of the nitrogen nucleus induces a moderately efficient spin relaxation [5]. The NMNA compound, the N-H peak was appeared at $\delta$ $4.65 \mathrm{ppm}$. In the case of NMNAIPA compound shows the $\mathrm{N}-\mathrm{H}$ peak appeared at $\delta 3.36 \mathrm{ppm}$ in the aromatic amine moiety. The variation of chemical shift in the $-\mathrm{NH}$ proton can be explained on the basis of attachment of NMNA to $\mathrm{COOH}$ proton of isophthalic acid moiety through hydrogen atom and the second - $\mathrm{COOH}$ acidic group are not involved in NMNA linked. The peak appeared at $\delta 2.51 \mathrm{ppm}$ is corresponding to DMSO solvent. The above spectral details clearly indicated to the formation of adduct compound as NMNAIPA.

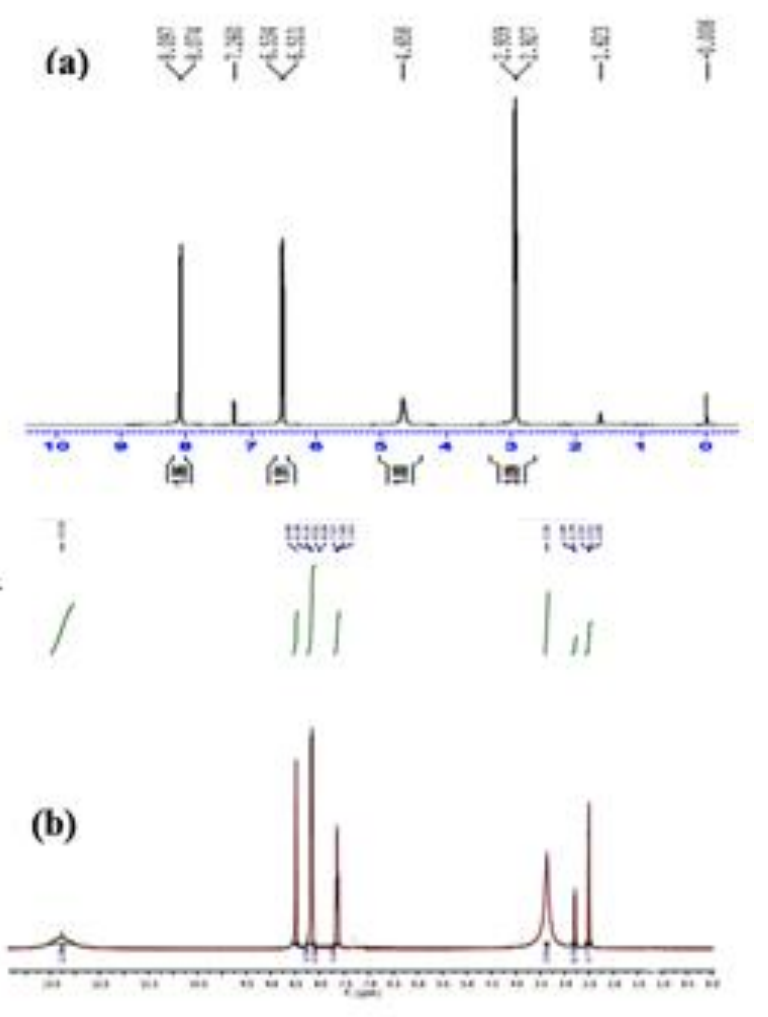

Fig .6. FT-NMR spectra of (a) N-methyl-4-nitroaniline (NMNA) and (b) NMNAIPA crystal

\section{E. ${ }^{13}$ C NMR spectral analysis}

The ${ }^{13} \mathrm{C}$ NMR spectrum of NMNAIPA is shown in Fig. 7. The NMNAIPA compound shows peak at $\delta 167.07 \mathrm{ppm}$ is due to the presence of carbonyl $(\mathrm{C}=\mathrm{O})$ carbon from acidic moiety. The peaks appeared at $\delta 156.73,136.92,133.86$, $131.68,130.43,129.64,126.29$ and $\delta 110.01 \mathrm{ppm}$, which is corresponds to aromatic carbons of benzene rings. The peak appeared at $\delta 29.86 \mathrm{ppm}$ in the up field region owes to methyl carbon in the N-methyl-4-nitroaniline moiety. 


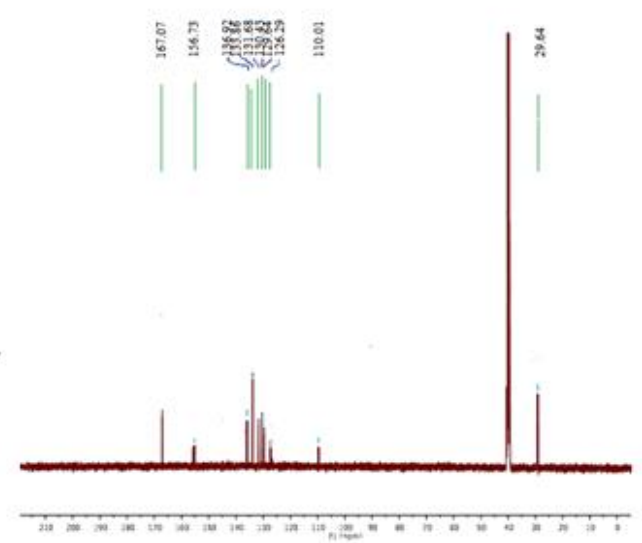

Fig. 7. ${ }^{13} \mathrm{C}$ NMR spectrum of NMNAIPA grown crystal

\section{F. UV-Vis- Spectral studies}

Solid state UV-Vis spectra of the parent N-methyl-4nitroaniline (NMNA) and NMNAIPA grown crystals was recorded in the wave length range between 200 and 1200 nm. Fig. 8 shows the plot of transmittance vs wavelength. The parent compound NMNA spectrum shows nearly $82 \%$ transmission with lower cutoff wavelength of $490 \mathrm{~nm}$ and the calculated band gap value is $2.53 \mathrm{eV}$. In the case of NMNAIPA crystal exhibit a transmittance of nearly $74 \%$ in wide wavelength range from 700 to $1000 \mathrm{~nm}$. The lower cut off wave length is observed at $436 \mathrm{~nm}$ and the band gap value is $2.75 \mathrm{eV}$. The wave length variation of absorption range was confirmed to the formation of NMNAIPA compound. The colored compounds absorb UV visible light generally with a strong absorbance in the visible range. The good transmittance property of the crystal in the entire visible region ensures its suitability for second harmonic generation application.

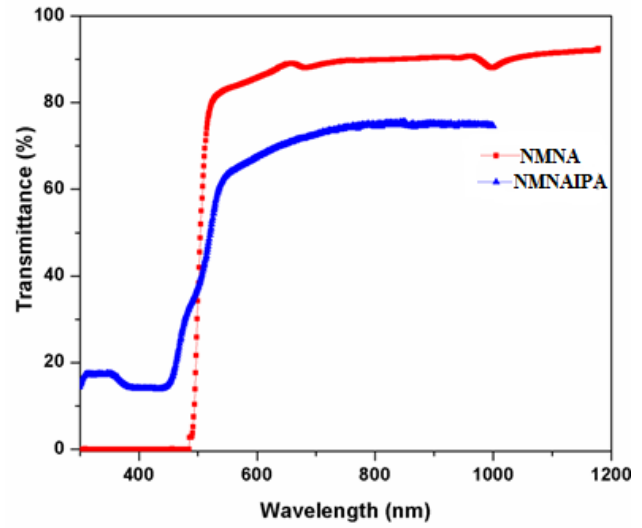

Fig. 8. Optical transmittance spectrum of NMNA and NMNAIAP crystals

\section{G. Thermal analysis}

The thermal behavior of growing crystal was studied by the thermo gravimetric analysis (TGA) and differential scanning calorimetric analysis (DSC). These characterizations techniques used to study of the phase transition, different stages of decomposition and thermal stability of the synthesized grown crystals. Fig.9 shows that TGA and DSC curve of NMNA and NMNAIPA compounds respectively. From the TGA curve of NMNA shows single stage of weight loss occur and it was thermal stability up to $403 \mathrm{~K}$. But in the case of NMNAIPA, it's seen that the two stages of consecutive mass losses. The first stage has minute weight loss occurred in $3.545 \%$ at $149^{\circ} \mathrm{C}$ of NMNAIPA compound. It is followed by major weight loss takes place in the temperature ranges from $241^{\circ} \mathrm{C}$ to $308^{\circ} \mathrm{C}$ with the total weight loss about $95.88 \%$ in the second stage. This mass loss suggests the elimination of nitro and carbon dioxide molecules and it's clear that the compound was decomposed at above the temperatures. Hence, compound decomposition temperatures were beyond $100^{\circ} \mathrm{C}$ and these indicates that no water of crystallization present in NMNAIPA compound. The TGA curve of NMNAIPA shows that the material is highly stable up to $421 \mathrm{~K}$. From the DSC curve of NMNAIPA compound show that strong endothermic peak appeared at $308^{\circ} \mathrm{C}$ respectively. These peaks are corresponding weight loss in TGA curve, which is attributed to the energy required for the release of the nitro and carbon dioxide molecules. The endothermic peaks coincide well with the decomposition observed in TGA trace. The DSC analysis also confirms the absence of physically adsorbed solvent or water of crystallization and its closely matching with TGA trace. Thus, the TGA and DSC analysis confirm the stability of the material, and which makes a potential material for laser applications and fabrication of any optical devices below its melting point.
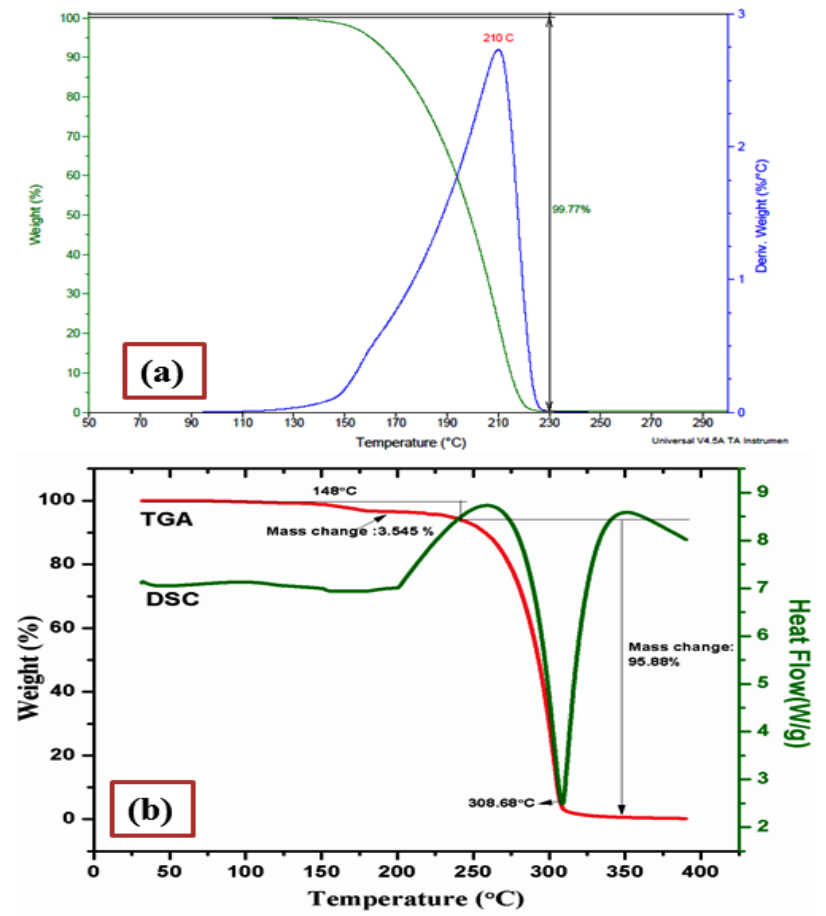

Fig. 9. TGA -DSC thermogram of (a) N-methyl-4-nitroaniline (NMNA) and (b) NMNAIPA compounds

\section{H. Dielectric studies}

The study of the dielectric characteristics of the material is most significant to conclude the lattice dynamics and transport occurrence in the crystal. It also covers the way to get the evidence about the nature of atoms, polarization mechanism, ions and bonding in the material [6]. Dielectric measurements were carried out grown NMNAIPA crystal using a frequency range is $50 \mathrm{~Hz}$ to $5 \mathrm{MHz}$ at different temperature ranging from 333,353 and $373 \mathrm{~K}$. Fig. 10 shows the variation of the dielectric constant with log frequency at different temperatures. From the plot, it was observed that the dielectric constant of NMNAIPA is high at low frequencies and it rapidly decreases with increasing frequency and becomes almost constant for higher 
frequencies, for all temperatures. As the net polarization present in the material is due to ionic polarization, electronic polarization, dipolar polarization and space charge polarization [6]. Ionic polarization, electronic polarization does not decrease very significantly with rise in temperature. The contribution of decrease in dielectric constant due to electronic polarization is very less. Dipolar polarization is also expected to decrease with temperature as it is inversely proportional to temperature. The contribution to polarizability to space charge depends on purity and perfection of crystals [7]. Fig. 11 shows the variation of the dielectric loss with log frequency at different temperatures. The dielectric loss also decreases with increasing frequency. The high value of dielectric loss at low frequencies is due to the oscillation of dipoles and at higher frequencies, all the polarizations are not operative. So no energy is consumed to rotate dipoles hence dielectric loss is high.

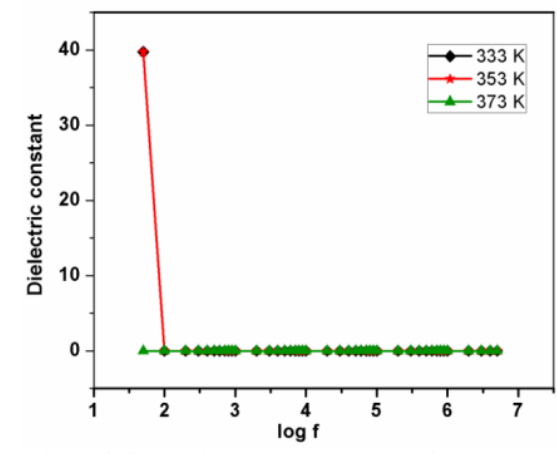

Fig. 10. Variation of dielectric constant with log frequency of NMNAIPA grown crystal

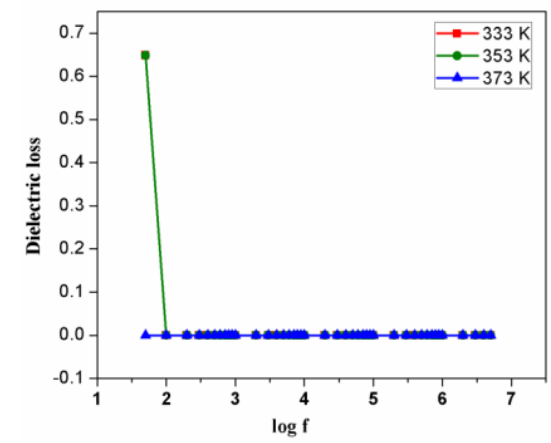

Fig. 11. Variation of dielectric loss with log frequency of NMNAIPA grown crystal

\section{Non-linear optical studies}

The second harmonic generation (SHG) property of grown crystalline powder of NMNAIPA was examined through modified Kurtz and Perry technique [7]. The NMNAIPA crystal was ground into powder to particle size 100-150 $\mu \mathrm{m}$. The powder NMNAIPA were tightly packed in a micro capillary tube mounted in the path of the QSwitched Nd: YAG laser was used as a light source. A fundamental laser beam of $1064 \mathrm{~nm}$ wavelength, pulse width $0.1 \mathrm{~s}$ with $10 \mathrm{~Hz}$ repetition rate was made to fall normally on a sample. The input energy used as $300 \mathrm{~mJ} /$ pulse, photomultiplier tube was actively as detector and oscilloscope assembly detected the green light emitted by the sample. The second harmonic signal $(\lambda=532)$ outputs are $50 \mathrm{mV}$ is obtained for NMNAIPA sample. The reference material KDP shows $40 \mathrm{mV}$ of the same frequency region. The SHG conversion efficiency of NMNAIPA crystal was divulged to be 1.25 times greater than that of reference material.

\section{CONCLUSION}

The new organic nonlinear optical material N-methyl-4nitroaniline isophthalic acid (NMNAIPA) adduct was synthesized and the single crystals were grown by adopting the solvent evaporation technique at ambient temperature. FT-IR, Raman and NMR spectral studies determine the molecular structure of growing crystal and also established the formation of molecular adduct. The UV-Vis NIR spectra show that NMNAI crystal transmittance nature in the visible region ensures its suitability for second harmonic generation application. From the TGA/DSC analysis shows that the thermal stability of growing crystal NMNAIPA found as $421 \mathrm{~K}$, the thermal stability desires its use in high temperature applications. Dielectric studies revealed that dielectric constant and dielectric loss was decreased with increasing frequency. The good dielectric behavior discovers a way to further advances required in the field of organic optoelectronic devices. The SHG test confirms the frequency doubling of the grown crystals and its exhibit NLO efficiency. The SHG conversion efficiency of NMNAIPA crystal was revealed to be 1.25 times greater than that of reference material KDP.

\section{REFERENCES}

[1] P. David, Shoemaker, Jerry Donohuem, Verner Schomaker, Robert B Corey, "The Crystal Structure of Ls-Threonine."J. Am. Chem.Soc. Vol. 72(6), pp. 2328-2349,1950.

[2] B.B. Ivanova, M. Spiteller, J. Phys. Chem. A,114, No. 15, pp. 5099 5103,2010.

[3] Irena Matulkova, Ivan Nemec, Karel Teubner, Petr Nemec, Zdenek Micka, Novel compounds of 4-amino-1,2,4-triazole with dicarboxylicacids - crystal structures, vibrational spectra and nonlinear optical properties, J. Mol. Struct., 873, pp. 46-60,2008.

[4] H. Hobart Willard, L. Lynne, Merritt Jr.,A.John, Dean, and A. Frank, Settle Jr., 'Instrumental methods of analysis', CBS Publishers and distributors, New Delhi, 1986.

[5] R. Silverstein, G.C. Bassler, T.C. Morrill, Spectrometric Identification of Organic Compounds, John Wiley \& Sons, 1981.

[6] M.A. Omar, Elementary Solid State Physics: Principles and Applications, Addison-Wesley, Reading, MA, pp.372- 408, 1975.

[7] S.K. Kurtz, and T.T. Perry, A powder technique for the evaluation of Nonlinear optical materials, J. App. Physics, 39,pp.3798-3813,1968.

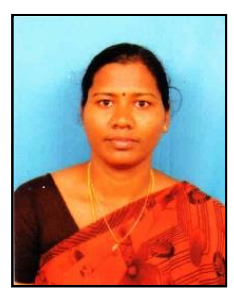

Lakshmi was born in Tamil Nadu State India and obtained B.Sc. Chemistry in University of Madras and M.Sc., M.Phil. Degrees in Chemistry from the Annamalai University, India in 2005 and 2009 respectively. She is currently a $\mathrm{PhD}$ student the Muthurangam Government Arts College, Vellore, India. Her research interest is in Crystal growth of organic non-linear optical materials.

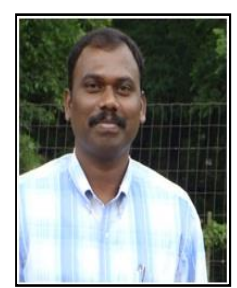

Dr. P. Prabukanthan is currently working as an Assistant Professor in the Department of Chemistry, Muthurangam Government Arts College, Vellore, India. He received his $\mathrm{Ph} . \mathrm{D}$ degree from Crysta Growth Centre, Anna University in 2008 and worked as a postdoctoral fellow at Department of Electrical Engineering, University of Nebraska-Lincoln, Lincoln, US from January 2008 to September 2008. His current research interests include heterojunction solar cells and organic non-liner optical materials. 